\title{
A!
}

This is an electronic reprint of the original article.

This reprint may differ from the original in pagination and typographic detail.

Lu, Bo; Burset, Pablo; Tanaka, Yukio

\section{Spin-polarized multiple Andreev reflections in spin-split superconductors}

Published in:

Physical Review B

DOI:

10.1103/PhysRevB.101.020502

Published: 08/01/2020

Document Version

Publisher's PDF, also known as Version of record

Please cite the original version:

Lu, B., Burset, P., \& Tanaka, Y. (2020). Spin-polarized multiple Andreev reflections in spin-split superconductors.

Physical Review B, 101(2), 1-6. [020502]. https://doi.org/10.1103/PhysRevB.101.020502

This material is protected by copyright and other intellectual property rights, and duplication or sale of all or part of any of the repository collections is not permitted, except that material may be duplicated by you for your research use or educational purposes in electronic or print form. You must obtain permission for any other use. Electronic or print copies may not be offered, whether for sale or otherwise to anyone who is not an authorised user. 


\title{
Spin-polarized multiple Andreev reflections in spin-split superconductors
}

\author{
Bo Lu $\odot,{ }^{1}$ Pablo Burset $\odot,{ }^{2}$ and Yukio Tanaka ${ }^{3}$ \\ ${ }^{1}$ Center for Joint Quantum Studies and Department of Physics, Tianjin University, Tianjin 300072, China \\ ${ }^{2}$ Department of Applied Physics, Aalto University, 00076 Aalto, Finland \\ ${ }^{3}$ Department of Applied Physics, Nagoya University, Nagoya 464-8603, Japan
}

(Received 9 October 2019; published 8 January 2020)

\begin{abstract}
We study the transport properties of a voltage-biased contact between two spin-split superconductors separated by an insulating barrier of arbitrary transparency. At low transparency, the contribution of multiple Andreev reflections leads to a subharmonic gap structure that crucially depends on the amplitude and relative angle of the spin-splitting fields of each superconductor. For noncollinear fields, we find an interesting even-odd effect on the bound states within the gap, where the odd order multiple Andreev reflections split, but the even order ones remain at their expected positions. By computing the current-voltage characteristics, we determine the transparency required for the emergence of a subharmonic gap structure and show that the splitting of the odd bound states is associated with different threshold energies of spin-polarized Andreev processes. Our findings provide a tool to experimentally determine the amplitude and alignment of Zeeman fields in spin-split superconductors.
\end{abstract}

DOI: 10.1103/PhysRevB.101.020502

Introduction. Hybrid structures between superconductors and magnetic materials reveal many interesting phenomena originated from the coexistence and interplay between ferromagnetism and superconductivity. As a result, the new field of superconducting spintronics has emerged [1,2], aiming at incorporating superconducting order into modern spintronic devices. The creation of spin-triplet Cooper pairs and spinpolarized quasiparticles with long spin-coherence lengths $[3,4]$ suggest future applications based on a reliable and efficient manipulation of spin-polarized currents [5,6]. In this context, superconductors with spin-split energy bands, commonly known as spin-split (or Zeeman-split) superconductors (SSc), are attracting considerable interest [7]. SSc can be realized either in a thin ferromagnet-superconductor (FM-S) junction via proximity effect [8-11] [cf. Fig. 1(a)], or in a thinfilm superconductor subject to a parallel (in-plane) magnetic field [12-15]. Highly spin-polarized currents can be generated in SSc hybrid junctions $[4,11,16]$ and large thermoelectric effects have been predicted [7,17-21].

The transport properties of hybrid junctions involving SSc has thus become a topic of fundamental interest in superconducting spintronics. For example, the phase difference in Josephson junctions between SSc has been used as a source to manipulate the spin-polarized supercurrents [9,22-25]. Recently, the tunneling quasiparticle current between two SSc linked by a spin-polarized barrier has been analyzed using quasiclassical Green's function techniques, showing good agreement between theory and experiments [26]. Here, we go one step further and analyze voltage-biased SSc Josephson junctions of arbitrary transparency, where multiple Andreev reflections (MAR) play an important role, and find that the subharmonic gap structure (SGS) is very sensitive to the spin-splitting fields of the superconductors.

When two superconductors are in electric contact, MAR [27-29] take place at voltages $e V<2 \Delta$, where $\Delta$ is the energy gap. In a symmetric junction involving conventional superconductors, electrons or holes can undergo sequential Andreev reflections at the interface. Due to the voltage bias, the quasiparticles will gain or lose an energy $e V$ as they travel across the interface, until escaping to the reservoirs for energies above the superconducting gap. This phenomenon results in the so-called SGS, a series of resonant conductance peaks at voltages $V_{n}=2 \Delta /(e n)$ in the current-voltage characteristics, where $n$ is an integer [27-32]. The peaks reveal the singular density of states at the superconducting energy gap edges [see Figs. 1(b)-1(e)]. The study of the SGS has proven to be useful for identifying properties of high- $T_{c}$ superconductors [33,34], topological superconductors $[35,36]$, and other mesoscopic hybrid junctions [37-43].

In this Rapid Communication, we study the transport properties in the MAR regime of a junction between two SSc, as depicted in Fig. 1(a). For simplicity, we only consider junctions where both superconductors have the same pair amplitude, $\Delta$, and strength of the spin-splitting field, $h$. However, we allow the junction to be asymmetric by changing the relative orientation, $\alpha$, of the in-plane spin-splitting fields. Without including phonon-induced spin flip [44], we find that the SGS due to MAR is highly tunable and presents a spin-dependent shift proportional to the Zeeman field $h$. Additionally, depending on the relative orientation of the spinsplitting fields, we find an even-odd effect on the conductance peaks forming the SGS. Figure 1 illustrates how MAR are modified by the spin-splitting fields. For $n$-order MAR, a quasiparticle undergoes $n-1$ Andreev reflections when transferring through the junction, and its spin is conserved since we are dealing with spin-singlet pairing states. Importantly, while MAR processes with odd order ( $n$ an odd integer) involve quasiparticles transferred between superconductors, for even order processes ( $n$ an even integer), quasiparticles instead return to the same superconductor. Therefore, only odd order MAR are sensitive to the relative orientation of the splitting fields. The threshold energies for quasiparticles traveling from 
(a)
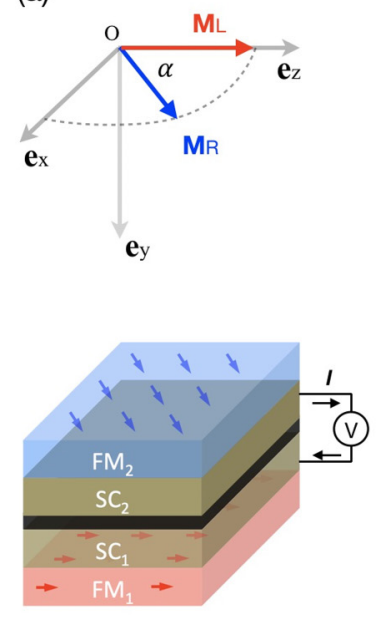

(b) $\quad \alpha=0, \mathrm{n}=3$

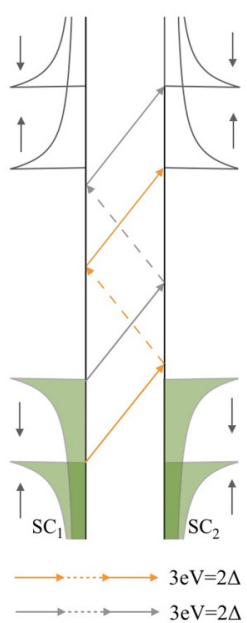

(c)

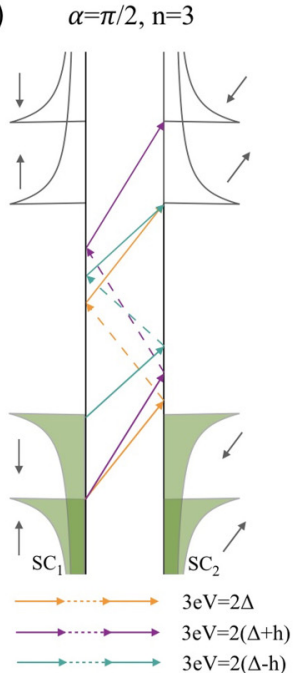

(d) $\alpha=\pi, \mathrm{n}=3$

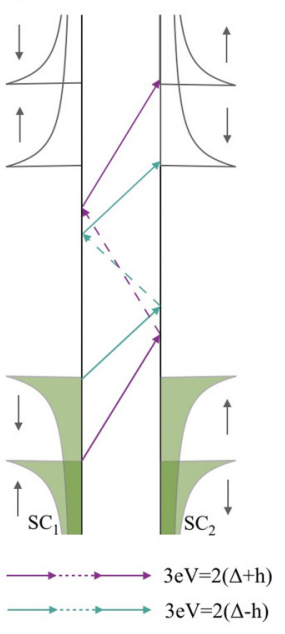

(e) $\alpha \in[0,2 \pi), \mathrm{n}=2$

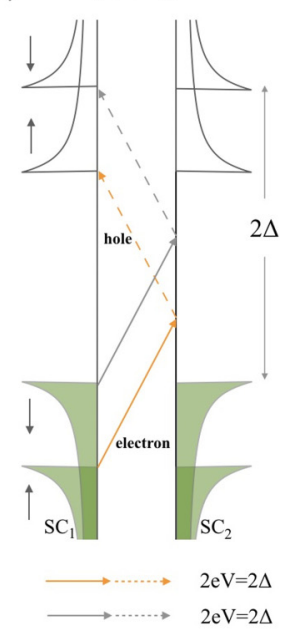

FIG. 1. (a) Schematic diagram of the multilayer FM-S-I-S-FM device used to develop a SSc-I-SSc Josephson junction, where I is an insulating barrier. The moments $\mathbf{M}_{L, R}$ of the FMs are confined to the $x$ - $z$ plane, with a relative angle $\alpha$, while transport takes place along the $y$ direction. (b)-(d) Schematic representation of the third-order MAR. The superconducting gap is $\Delta$ and the energy bands are split by the Zeeman fields. Solid (dashed) lines represent electron (hole) trajectories. The relative angle $\alpha$ affects the positions of the threshold voltages for odd order MAR. (e) Second-order MAR, with threshold voltage at $e V=\Delta$, is independent of $\alpha$.

one side to the other are $2 \Delta$ for parallel magnetization $(\alpha=0)$ and $2(\Delta \pm h)$ for antiparallel magnetization $(\alpha=\pi)$. Arbitrary values of $\alpha$ lead to spin mixing and result in three possible channels $2 \Delta$ and $2(\Delta \pm h)$. Thus, the odd subharmonics become $2 \Delta / n$ for $\alpha=0$ [see Fig. 1(b)], $2(\Delta \pm h) / n$ for $\alpha=$ $\pi$ [see Fig. 1(d)], and $2 \Delta / n, 2(\Delta \pm h) / n$ for $\alpha \neq 0, \pi$ [see Fig. 1(c)]. However, for even order processes, quasiparticles travel back to the same side, with spin conserved, and thus the threshold energy gains or losses between the two gap edges are independent of the splitting field, as shown in Fig. 1(e). Therefore, the position of the conductance peaks for even subharmonics, $2 \Delta / n$, is not altered by the spin-splitting fields. The SGS thus provides useful information about the strength and relative orientation of the spin-splitting fields at Josephson junctions between SSc.

Model. The system we study consists of two semi-infinite superconductors in a point contact geometry. Spin-splitting fields are induced on both sides via proximity effect to a FM region. The phenomenological Hamiltonian of the system is written as $\hat{H}=\hat{H}_{L}+\hat{H}_{R}+\hat{H}_{T}(t) . \hat{H}_{L}$ and $\hat{H}_{R}$ describe the bulk SSc on the left and right side:

$$
\hat{H}_{j=L, R}=\frac{1}{2} \sum_{k} \hat{\Psi}_{j k}^{\dagger}\left[\begin{array}{cc}
H_{j k} & \hat{\Delta} \\
\hat{\Delta}^{\dagger} & -H_{j k}^{T}
\end{array}\right] \hat{\Psi}_{j k},
$$

where $\hat{\Psi}_{j k}=\left[\psi_{j k, \uparrow}, \psi_{j k, \downarrow}, \psi_{j(-k), \uparrow}^{\dagger}, \psi_{j(-k), \downarrow}^{\dagger}\right]^{T}$ is the spinor in Nambu-spin space. The noninteracting Hamiltonian is

$$
H_{j k}=\left[k^{2} /(2 m)-\mu\right] \hat{\sigma}_{0}-g \mu_{B} \hat{\sigma} \cdot \mathbf{M}_{j}
$$

We assume that the orientation of the Zeeman fields lies in the $x-z$ plane and parametrize the moments as $\mathbf{M}_{L}=M \mathbf{e}_{z}$ and $\mathbf{M}_{R}=M\left[\sin \alpha \mathbf{e}_{x}+\cos \alpha \mathbf{e}_{z}\right]$, with $g, \mu_{B}$, and $\mathbf{M}_{L, R}$ the effective Landé $g$ factor, Bohr magneton, and induced magnetic fields, respectively. Other spin orientations can be accounted for by an appropriate rotation without affecting our results. The gap matrix in Eq. (1) is $\hat{\Delta}=i \hat{\sigma}_{y} \Delta$ for spin-singlet $s$-wave pairing, where the Pauli matrices $\hat{\sigma}_{0, x, y, z}$ operate in spin space. The tunneling term $\hat{H}_{T}(t)$ is given by

$$
\hat{H}_{T}(t)=\frac{1}{2} \sum_{k, k^{\prime}}\left[\hat{\Psi}_{L k}^{\dagger} \hat{T}_{k k^{\prime}}(t) \hat{\Psi}_{R k^{\prime}}+\text { H.c. }\right],
$$

with $\hat{T}_{k k^{\prime}}(t)=t_{k k^{\prime}} \hat{\tau}_{z} e^{i \chi(t) \hat{\tau}_{z} / 2}$ and $\hat{\tau}_{x, y, z}$ the Pauli matrices in particle-hole space. In the presence of a voltage bias $V$, the superconducting phase difference $\chi(t)=2 \omega_{J} t$ is time dependent, with $\omega_{J}=2 \mathrm{eV}$ the Josephson frequency. The resulting time-dependent current follows the Josephson relation $\Delta \rightarrow \Delta e^{i 2 e V t}$ [45], with $I(t)=\sum_{n} I_{n} e^{i n \omega_{J} t}$. For simplicity, we set $t_{k k^{\prime}}$ to be $t_{k k^{\prime}}=t_{0} \delta_{k, k^{\prime}}$ and denote $\hat{T}(t)=t_{0} \hat{\tau}_{z} e^{i \chi(t) \hat{\tau}_{z} / 2}$. Following the quasiclassical approximation [46,47], we average the summation over channels $k$ without affecting the characteristics of the SGS, but simplifying calculations. By the so-called $\xi$ integration, the retarded/advanced Green's function $\hat{\mathcal{G}}_{j}^{r / a}(\omega)$ in the bulk state adopts the form [48]

$$
\hat{\mathcal{G}}_{L}^{r / a}=\left[\begin{array}{cccc}
g_{\uparrow}^{r / a} & 0 & 0 & f_{\uparrow}^{r / a} \\
0 & g_{\downarrow}^{r / a} & f_{\downarrow}^{r / a} & 0 \\
0 & f_{\downarrow}^{r / a} & g_{\downarrow}^{r / a} & 0 \\
f_{\uparrow}^{r / a} & 0 & 0 & g_{\uparrow}^{r / a}
\end{array}\right] \triangleq\left[\begin{array}{cc}
\stackrel{\Upsilon}{1}_{11}^{r / a} & \stackrel{\circ}{\Upsilon}_{12}^{r / a} \\
\stackrel{\Upsilon}{\Upsilon}_{21}^{r / a} & \stackrel{\circ}{\Upsilon}_{22}^{r / a}
\end{array}\right],
$$

where

$$
\begin{gathered}
\hat{\mathcal{G}}_{R}^{r / a}=\hat{U} \hat{\mathcal{G}}_{L}^{r / a} \hat{U}^{\dagger} \triangleq\left[\begin{array}{cc}
\stackrel{\circ}{\Gamma}_{11}^{r / a} & \stackrel{\circ}{\Gamma}_{12}^{r / a} \\
\stackrel{\circ}{\Gamma}_{21}^{r / a} & \stackrel{\circ}{\Gamma}_{22}^{r / a}
\end{array}\right], \\
g_{\sigma}^{r / a}(\omega)=\frac{-\left(\omega+\zeta_{\sigma} h \pm i 0^{+}\right)}{W \sqrt{\Delta^{2}-\left(\omega+\zeta_{\sigma} h \pm i 0^{+}\right)^{2}}} \\
f_{\sigma}^{r / a}(\omega)=\frac{\Delta}{W \sqrt{\Delta^{2}-\left(\omega+\zeta_{\sigma} h \pm i 0^{+}\right)^{2}}}
\end{gathered}
$$



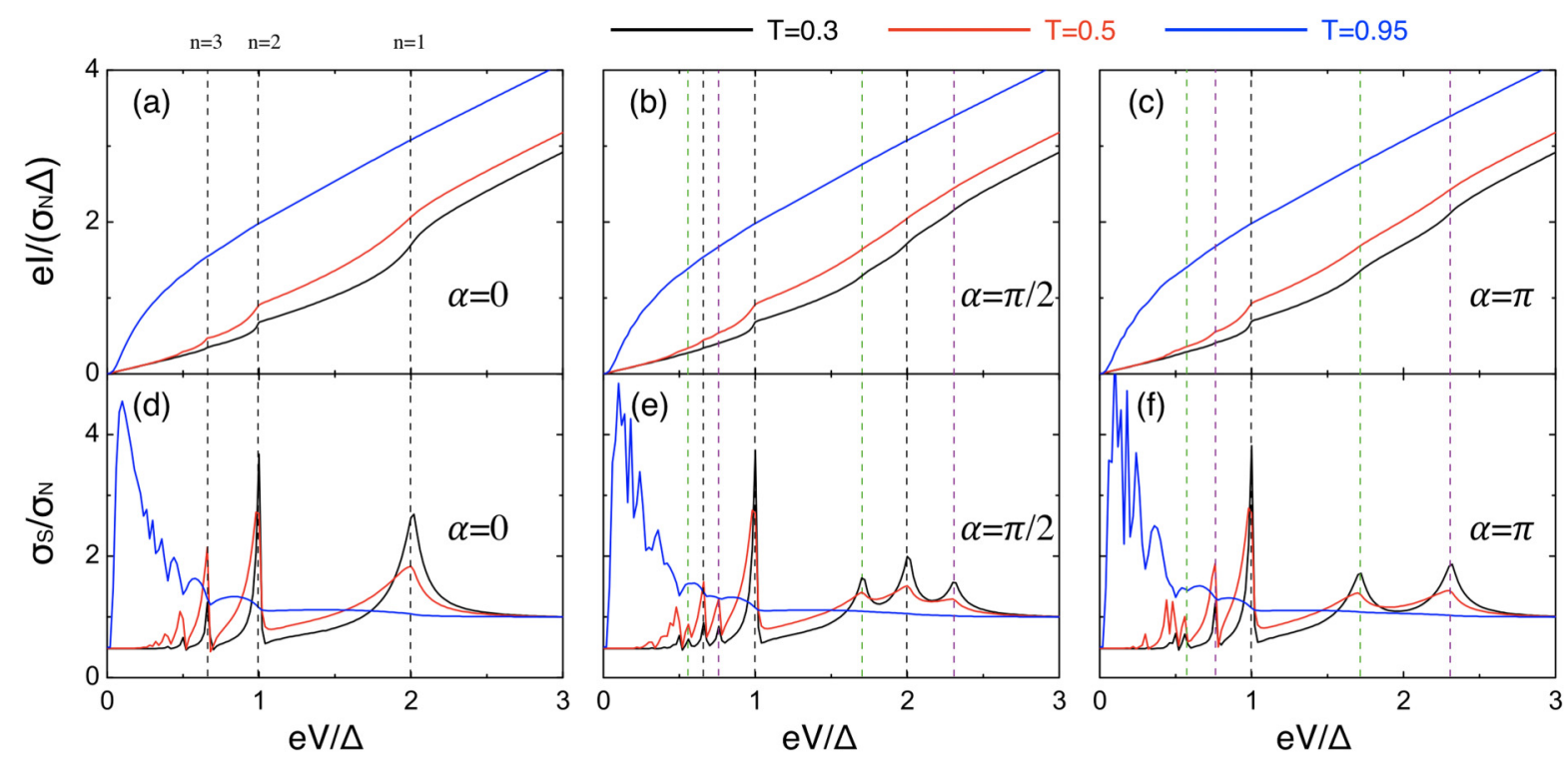

FIG. 2. Electric current and differential conductance as a function of the voltage, for relative angles $\alpha=0, \pi / 2, \pi$, and transmissions $T=0.95,0.5,0.3$. In all cases, $h / \Delta=0.15$. The dashed vertical lines show the $n$ th-order SGS at $2 \Delta / n$ for $n=1,2,3$ (black), 2( $\Delta-h) / n$ for $n=1,3$ (green), and $2(\Delta+h) / n$ for $n=1,3$ (purple).

with $\zeta_{\sigma=\uparrow, \downarrow}= \pm 1$ and $\hat{U}=e^{-i \hat{\sigma}_{y} \alpha / 2} \hat{\tau}_{0}$. The magnitude of the proximity-induced spin-splitting fields is $h=g \mu_{B} M$ and $W$ is a band parameter [37]. We choose the magnetic field below the so-called Chandrasekhar-Clogston limit [49,50], $h_{\max }=$ $\Delta_{0} / \sqrt{2}$. The Keldysh Green's function $\hat{\mathcal{G}}_{j=L, R}^{K}(\omega)$ is given by $\hat{\mathcal{G}}_{j}^{K}(\omega)=\left[\hat{\mathcal{G}}_{j}^{r}(\omega)-\hat{\mathcal{G}}_{j}^{a}(\omega)\right] \tanh \left[\omega /\left(2 k_{B} T\right)\right][51]$.

Following the transfer-matrix approach and double Fourier transformation $[32,37,52]$, we express the charge current in the following form:

$$
\mathcal{I}(t)=e v(0) v_{F} \sum_{m} \int \frac{d \omega}{8 \pi} \operatorname{Tr}\left[\hat{\tau}_{z} \mathcal{I}_{m}\right] e^{i m \omega_{J} t / 2},
$$

with $v(0)$ the density of states at the Fermi level in the normal state. The components $\mathcal{I}_{m}$ are defined as

$$
\begin{aligned}
\mathcal{I}_{m}= & \sum_{n} \hat{\mathcal{G}}_{R, 0}^{r}\left[\mathcal{T}_{n 0}^{a}\right]^{\dagger} \hat{\mathcal{G}}_{L, n}^{K} \mathcal{T}_{n m}^{a}+\mathcal{T}_{0 n}^{r} \hat{\mathcal{G}}_{R, n}^{K}\left[\mathcal{T}_{m n}^{r}\right]^{\dagger} \hat{\mathcal{G}}_{L, m}^{a} \\
& -\left[\mathcal{T}_{n 0}^{a}\right]^{\dagger} \hat{\mathcal{G}}_{L, n}^{K} \mathcal{T}_{n m}^{a} \hat{\mathcal{G}}_{R, m}^{a}-\hat{\mathcal{G}}_{L, 0}^{r} \mathcal{T}_{0 n}^{r} \hat{\mathcal{G}}_{R, n}^{K}\left[\mathcal{T}_{m n}^{r}\right]^{\dagger},
\end{aligned}
$$

with $\hat{\mathcal{G}}_{j, n}^{r, a, K}=\hat{\mathcal{G}}_{j}^{r a, K}\left(\omega+n \omega_{J} / 2\right)$. The dc component of the current corresponds to the $m=0$ harmonic in Eq. (8). Experimentally, it relates to the average electric current in the long-time limit. The transfer matrix satisfies $\mathcal{T}_{n m}^{r / a}(\omega)=$ $\mathcal{T}_{n-m, 0}^{r / a}\left(\omega+m \omega_{J} / 2\right)$ and can be determined by the recursive relation

$$
\begin{aligned}
\hat{\mathcal{T}}_{n m}^{r / a}= & t_{0}\left[\begin{array}{cc}
\hat{\sigma}_{0} & \mathbf{0} \\
\mathbf{0} & \mathbf{0}
\end{array}\right] \delta_{n,-1}+t_{0}\left[\begin{array}{cc}
\mathbf{0} & \mathbf{0} \\
\mathbf{0} & -\hat{\sigma}_{0}
\end{array}\right] \delta_{n, 1} \\
& +\hat{\epsilon}_{n}^{r / a} \hat{\mathcal{T}}_{n m}^{r / a}+\hat{V}_{n, n+2}^{r / a} \hat{\mathcal{T}}_{n+2, m}^{r / a}+\hat{\Lambda}_{n, n-2}^{r / a} \hat{\mathcal{T}}_{n-2, m}^{r / a},
\end{aligned}
$$

with

$$
\begin{aligned}
& \hat{\epsilon}_{n}^{r / a}=t_{0}^{2}\left[\begin{array}{llll}
\stackrel{\circ}{\Gamma}_{11, n+1}^{r / a} & \stackrel{\circ}{\Upsilon}_{11, n}^{r / a} & \stackrel{\circ}{\Gamma}_{11, n+1}^{r / a} & \stackrel{\circ}{\Upsilon}_{12, n}^{r / a} \\
\stackrel{\circ}{\Gamma}_{22, n-1}^{r / a} & \stackrel{\circ}{\Upsilon} & r / a & \circ \\
\Gamma_{21, n}^{r / a} & \stackrel{\circ}{2}_{22, n-1}^{r / a} &
\end{array}\right], \\
& \hat{V}_{n, n+2}^{r / a}=-t_{0}^{2}\left[\begin{array}{cc}
\stackrel{\circ}{\Gamma}_{12, n+1}^{r / a} \stackrel{\circ}{\Upsilon}_{21, n+2}^{r / a} & \stackrel{o}{\Gamma}_{12, n+1}^{r / a} \stackrel{\circ}{\Upsilon}_{22, n+2}^{r / a} \\
0 & 0
\end{array}\right] \text {, }
\end{aligned}
$$

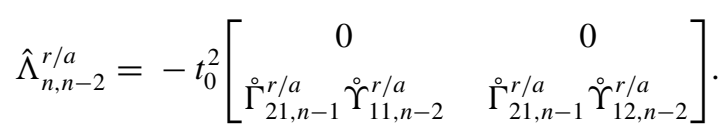

Equation (10) is numerically solved by introducing ladder operators $\mathcal{T}_{n, m}^{r / a}=z_{n, \pm}^{r / a} \mathcal{T}_{n \mp 2, m}^{r / a}$ and using cut-off values $z_{n, \pm}^{r / a}=0$ for a sufficient large $|n|=n_{N}$, where $\mathcal{T}_{ \pm n_{N}, 0}^{r / a}$ are assumed to vanish. We normalize the current in units of $\sigma_{N} \Delta / e$, where $\sigma_{N}$ is the conductance when both electrodes are in nonsuperconducting states without magnetic elements.

Josephson dc current. We now compute the current-voltage characteristics of a dc-biased contact between two SSc. As explained before, we set the gap of both superconductors to be the same, $\Delta$, and the spin-splitting field is also equal, $h$. We show in Fig. 2 the current and differential conductance, $\sigma_{\mathrm{S}}$, for different values of the transmission, $T$, and relative orientation angle, $\alpha$. In the case of a high-transmission barrier, i.e., $T \lesssim 1$, the current looks almost featureless, but its derivative displays small peaks due to MAR, which are more prominent at low voltages (blue lines in Fig. 2). This is a result of the enhanced probability for Andreev reflections at high transmissions. For lower transmissions, $T \lesssim 0.5$, the subgap Andreev reflections are suppressed and thus the SGS becomes more visible as kinks in the current and peaks in the differential conductance (red and black lines in Fig. 2). These features are now more 

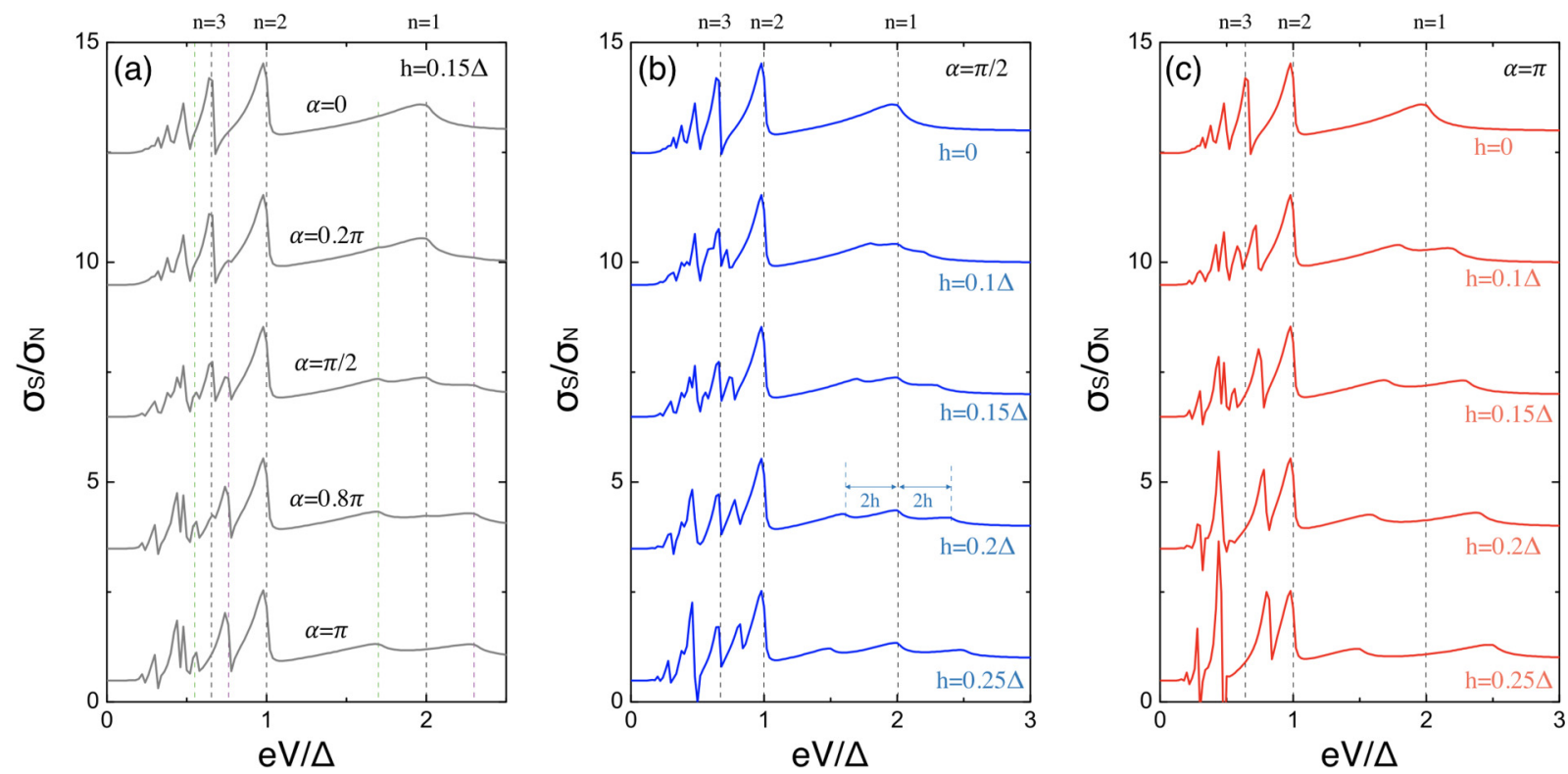

FIG. 3. Normalized differential conductance as a function of the voltage. (a) Plot of the conductance for several relative angles, with fixed $h / \Delta=0.15$. (b), (c) Plot of the conductance for various spin-splitting fields, with fixed $\alpha=\pi / 2$ for (b) and $\alpha=\pi$ for (c). In all cases, the transmission is fixed at $T=0.6$ and the curves have been vertically displaced for clarity. The dashed vertical lines show the $n$ th-order SGS at $2 \Delta / n$ for $n=1,2,3$ (black), $2(\Delta-h) / n$ for $n=1,3$ (green), and $2(\Delta+h) / n$ for $n=1,3$ (purple).

visible at higher voltages, due to the quasiparticle transfer at the gap edges in the tunneling regime. These are common features of MAR in superconducting junctions; the novel effect comes from the angle $\alpha$ determining the relative orientations of the spin-splitting fields. Figure 2 shows the three representative cases with $\alpha=0, \pi$ (parallel and antiparallel) and $\alpha \neq 0, \pi$ (noncollinear). As observed in Figs. 2(a) and 2(d), where the fields are parallel, the SGS shows the usual distribution with peaks at $e V=2 \Delta / n$. This result is similar to previous works [32] without induced spin-split field in superconductors. By contrast, when the fields are antiparallel, the odd order MAR at $e V=2 \Delta / n$ disappear while new peaks at $e V=2(\Delta \pm h) / n$ emerge ( $n$ is an odd integer) [see Figs. 2(c) and 2(f)]. Finally, when the magnetic field is noncollinear, the odd order MAR feature both types of peaks, at $e V=2 \Delta / n$ and at $e V=2(\Delta \pm h) / n$, as shown in Figs. 2(b) and 2(e). Importantly, the splitting of the odd order peaks for $\alpha \neq 0$ is directly proportional to the amplitude of the Zeeman field $h$.

From this result, we conclude that the odd SGS are very sensitive to the configuration of the two spin-split fields. Thus, the $I-V$ characteristics could provide a way to measure the amplitude and alignment of the spin-splitting fields. To clearly show these dependences on the spin-split fields, we plot the normalized differential conductance for a junction with average transmission $T=0.6$ in Fig. 3. First, we fix the magnitude of $h$ and change the relative angle $\alpha$ in Fig. 3(a). The odd order MAR split into three peaks when $\alpha \neq 0$. The peaks at $e V=2(\Delta \pm h) / n$ reach their maximum as $\alpha$ approaches $\pi$. By contrast, the peaks at $e V=2 \Delta / n$ are significantly reduced until they disappear for $\alpha=\pi$. Importantly, the even order MAR remain unchanged as $\alpha$ varies. Next, we show in Figs. 3(b) and 3 (c) the SGS for several values of $h$ in the representative cases of noncollinear $(\alpha=\pi / 2)$ and antiparallel $(\alpha=\pi)$ fields. This result indicates the great tunability of the SGS by both the relative angle and the magnitude of spin-split field. Moreover, the tunability is attributed to the spin polarization of odd order MAR. The splitting of the odd order MAR becomes wider as $h$ increases. It is noted that we find no asymmetry in the $d I / d V$ curve with respect to the sign of $V$ since we have no middle spin-filter barrier [26]. The additional spin mixing induced by a spin-filter barrier could result in the splitting of the even order MAR.

Conclusions. We have theoretically analyzed the SGS in a Josephson junction between two spin-split superconductors with arbitrary amplitude and orientation of their Zeeman fields. Our results show an interesting even-odd effect of the bound states within the gap. The SGS induced by odd MAR are split by the Zeeman fields and they are strongly influenced by their relative angle. The SGS from even MAR, instead, are independent of the spin-splitting fields. The analysis of the SGS is thus a useful tool to determine with great precision the magnitude and relative orientation of the Zeeman fields in experiments. We remark that, although we consider a simplified single-channel superconducting weak link model, the splitting of odd-order MAR resonances is also present in common setups between spin-split superconductors, such as in a two-dimensional planar junction.

Acknowledgments. We thank J. Cayao, S. Suzuki, S. Tamura, and A. Yamakage for insightful discussions. We acknowledge support from the National Natural Science Foundation of China (project 11904257); the Horizon 2020 Research and Innovation Programme under the Marie Skłodowska-Curie Grant No. 743884 and the Academy of Finland (project 312299); Topological Material Science (Grants No. JP15H05851, No. JP15H05853, and No. JP15K21717); and Grant-in-Aid for Scientific Research B (Grant No. JP18H01176) from the Ministry of Education, Culture, Sports, Science, and Technology, Japan (MEXT). 
[1] J. Linder and J. W. A. Robinson, Superconducting spintronics, Nat. Phys. 11, 307 (2015).

[2] M. Eschrig, Spin-polarized supercurrents for spintronics: A review of current progress, Rep. Prog. Phys. 78, 104501 (2015).

[3] H. Yang, S.-H. Yang, S. Takahashi, S. Maekawa, and S. S. P. Parkin, Extremely long quasiparticle spin lifetimes in superconducting aluminium using $\mathrm{MgO}$ tunnel spin injectors, Nat. Mater. 9, 586 (2010).

[4] F. Hübler, M. J. Wolf, D. Beckmann, and H. v. Löhneysen, Long-Range Spin-Polarized Quasiparticle Transport in Mesoscopic Al Superconductors with a Zeeman Splitting, Phys. Rev. Lett. 109, 207001 (2012).

[5] D. Breunig, P. Burset, and B. Trauzettel, Creation of SpinTriplet Cooper Pairs in the Absence of Magnetic Ordering, Phys. Rev. Lett. 120, 037701 (2018).

[6] K. Halterman, O. T. Valls, and M. Alidoust, Spin-Controlled Superconductivity and Tunable Triplet Correlations in Graphene Nanostructures, Phys. Rev. Lett. 111, 046602 (2013).

[7] F. S. Bergeret, M. Silaev, P. Virtanen, and T. T. Heikkilä, Colloquium: Nonequilibrium effects in superconductors with a spin-splitting field, Rev. Mod. Phys. 90, 041001 (2018).

[8] P. M. Tedrow, J. E. Tkaczyk, and A. Kumar, Spin-Polarized Electron Tunneling Study of An Artificially Layered Superconductor with Internal Magnetic Field: EuO-Al, Phys. Rev. Lett. 56, 1746 (1986).

[9] F. S. Bergeret, A. F. Volkov, and K. B. Efetov, Long-Range Proximity Effects in Superconductor-Ferromagnet Structures, Phys. Rev. Lett. 86, 4096 (2001).

[10] F. S. Bergeret, A. F. Volkov, and K. B. Efetov, "Odd triplet superconductivity and related phenomena in superconductorferromagnet structures," Rev. Mod. Phys. 77, 1321 (2005).

[11] F. Giazotto and F. Taddei, Superconductors as spin sources for spintronics, Phys. Rev. B 77, 132501 (2008).

[12] R. Meservey, P. M. Tedrow, and P. Fulde, Magnetic Field Splitting of the Quasiparticle States in Superconducting Aluminum Films, Phys. Rev. Lett. 25, 1270 (1970).

[13] P. M. Tedrow and R. Meservey, Spin-Dependent Tunneling Into Ferromagnetic Nickel, Phys. Rev. Lett. 26, 192 (1971).

[14] P. M. Tedrow and R. Meservey, Spin polarization of electrons tunneling from films of Fe, Co, Ni, and Gd, Phys. Rev. B 7, 318 (1973).

[15] R. Meservey and P. M. Tedrow, Spin-polarized electron tunneling, Phys. Rep. 238, 173 (1994).

[16] J. Linder, T. Yokoyama, Y. Tanaka, and A. Sudbø, Strongly spin-polarized current generated in a Zeeman-split unconventional superconductor, Phys. Rev. B 78, 014516 (2008).

[17] M. S. Kalenkov, A. D. Zaikin, and L. S. Kuzmin, Theory of A Large Thermoelectric Effect in Superconductors Doped with Magnetic Impurities, Phys. Rev. Lett. 109, 147004 (2012).

[18] P. Machon, M. Eschrig, and W. Belzig, Nonlocal Thermoelectric Effects and Nonlocal Onsager Relations in A ThreeTerminal Proximity-Coupled Superconductor-Ferromagnet Device, Phys. Rev. Lett. 110, 047002 (2013).

[19] A. Ozaeta, P. Virtanen, F. S. Bergeret, and T. T. Heikkilä, Predicted Very Large Thermoelectric Effect in FerromagnetSuperconductor Junctions in the Presence of a Spin-Splitting Magnetic Field, Phys. Rev. Lett. 112, 057001 (2014).

[20] P. Machon, M. Eschrig, and W. Belzig, Giant thermoelectric effects in a proximity-coupled superconductor-ferromagnet device, New J. Phys. 16, 073002 (2014).
[21] F. Keidel, S.-Y. Hwang, B. Trauzettel, B. Sothmann, and P. Burset, On-demand thermoelectric generation of equal-spin Cooper pairs, arXiv:1907.00965.

[22] J. E. Tkaczyk and P. M. Tedrow, Magnetic proximity effect at a superconductor-rare-earth oxide interface, J. Appl. Phys. 61, 3368 (1987).

[23] X. Hao, J. S. Moodera, and R. Meservey, Thin-Film Superconductor in an Exchange Field, Phys. Rev. Lett. 67, 1342 (1991).

[24] Y. M. Xiong, S. Stadler, P. W. Adams, and G. Catelani, SpinResolved Tunneling Studies of the Exchange Field in EuS/Al Bilayers, Phys. Rev. Lett. 106, 247001 (2011).

[25] F. S. Bergeret, A. Verso, and A. F. Volkov, Spin-polarized Josephson and quasiparticle currents in superconducting spinfilter tunnel junctions, Phys. Rev. B 86, 060506(R) (2012).

[26] M. Rouco, S. Chakraborty, F. Aikebaier, V. N. Golovach, E. Strambini, J. S. Moodera, F. Giazotto, T. T. Heikkilä, and F. S. Bergeret, Charge transport through spin-polarized tunnel junction between two spin-split superconductors, Phys. Rev. B 100, 184501 (2019).

[27] T. M. Klapwijk, G. E. Blonder, and M. Tinkham, Explanation of subharmonic energy gap structure in superconducting contacts, Physica B+C 109-110, 1657 (1982), Part of special issue: 16th International Conference on Low Temperature Physics, Part 3.

[28] M. Octavio, M. Tinkham, G. E. Blonder, and T. M. Klapwijk, Subharmonic energy-gap structure in superconducting constrictions, Phys. Rev. B 27, 6739 (1983).

[29] G. B. Arnold, Superconducting tunneling without the tunneling Hamiltonian. II. Subgap harmonic structure, J. Low Temp. Phys. 68, 1 (1987).

[30] E. N. Bratus', V. S. Shumeiko, and G. Wendin, Theory of Subharmonic Gap Structure in Superconducting Mesoscopic Tunnel Contacts, Phys. Rev. Lett. 74, 2110 (1995).

[31] D. Averin and A. Bardas, ac Josephson Effect in a Single Quantum Channel, Phys. Rev. Lett. 75, 1831 (1995).

[32] J. C. Cuevas, A. Martín-Rodero, and A. Levy Yeyati, Hamiltonian approach to the transport properties of superconducting quantum point contacts, Phys. Rev. B 54, 7366 (1996).

[33] A. Poenicke, J. C. Cuevas, and M. Fogelström, Subharmonic gap structure in $d$-wave superconductors, Phys. Rev. B 65, 220510(R) (2002).

[34] J. C. Cuevas and M. Fogelström, Shot Noise and Multiple Andreev Reflections in $d$-Wave Superconductors, Phys. Rev. Lett. 89, 227003 (2002).

[35] D. M. Badiane, M. Houzet, and J. S. Meyer, Nonequilibrium Josephson Effect through Helical Edge States, Phys. Rev. Lett. 107, 177002 (2011).

[36] P. San-Jose, J. Cayao, E. Prada, and R. Aguado, Multiple Andreev reflection and critical current in topological superconducting nanowire junctions, New J. Phys. 15, 075019 (2013).

[37] J. C. Cuevas and M. Fogelström, Quasiclassical description of transport through superconducting contacts, Phys. Rev. B 64, 104502 (2001).

[38] I. V. Bobkova, Subharmonic gap structure in superconductor/ferromagnet/superconductor junctions, Phys. Rev. B 73, 012506 (2006).

[39] J. C. Cuevas and A. Levy Yeyati, Subharmonic gap structure in short ballistic graphene junctions, Phys. Rev. B 74, 180501(R) (2006). 
[40] I. V. Bobkova and A. M. Bobkov, Influence of spin filtering and spin mixing on the subgap structure of $I-V$ characteristics in a superconducting quantum point contact, Phys. Rev. B 76, 094517 (2007).

[41] F. Dolcini and L. Dell'Anna, Multiple Andreev reflections in a quantum dot coupled to superconducting leads: Effect of spinorbit coupling, Phys. Rev. B 78, 024518 (2008).

[42] S. Duhot, F. Lefloch, and M. Houzet, Cross Correlation of Incoherent Multiple Andreev Reflections, Phys. Rev. Lett. 102, 086804 (2009).

[43] C. Holmqvist, M. Fogelström, and W. Belzig, Spin-polarized Shapiro steps and spin-precession-assisted multiple Andreev reflection, Phys. Rev. B 90, 014516 (2014).

[44] J. Lang, W. Eisenmenger, and P. Fulde, Phonon-Induced Spin Flip in Extremely Thin Superconducting Al Tunneling Junctions in High Magnetic Fields, Phys. Rev. Lett. 77, 2546 (1996).

[45] B. D. Josephson, Supercurrents through barriers, Adv. Phys. 14, 419 (1965).

[46] B. Lu, P. Burset, Y. Tanuma, A. A. Golubov, Y. Asano, and Y. Tanaka, Influence of the impurity scattering on charge transport in unconventional superconductor junctions, Phys. Rev. B 94, 014504 (2016).

[47] P. Burset, B. Lu, S. Tamura, and Y. Tanaka, Current fluctuations in unconventional superconductor junctions with impurity scattering, Phys. Rev. B 95, 224502 (2017).

[48] J. Linder and J. W. A. Robinson, Strong odd-frequency correlations in fully gapped Zeeman-split superconductors, Sci. Rep. 5, 15483 (2015).

[49] B. S. Chandrasekhar, A note on the maximum critical field of high-field superconductors, Appl. Phys. Lett. 1, 7 (1962).

[50] A. M. Clogston, Upper Limit for the Critical Field in Hard Superconductors, Phys. Rev. Lett. 9, 266 (1962).

[51] The temperature dependence of the superconducting gap function is approximated as $\Delta(T)=\Delta_{0} \tanh \left(1.74 \sqrt{T_{c} / T-1}\right)$, with $\Delta_{0}=1.76 k_{B} T_{c}$ and $T_{c}$ the critical temperature. We take a sufficient low temperature $k_{B} T=10^{-3} \Delta_{0}$ in our calculation and set the band parameter $W=1$ without qualitatively influencing our results.

[52] S. Graser and T. Dahm, Transfer-matrix approach to multiband Josephson junctions, Phys. Rev. B 75, 014507 (2007). 